\title{
A REMARK ON A PAPER OF JI-SHOU
}

\author{
PATRICK M. FITZPATRICK AND SEYMOUR GOLDBERG
}

(Communicated by Palle E. T. Jorgensen)

\begin{abstract}
We observe that a strictly singular operator is not necessarily condensing, so that the invariant subspace problem for strictly singular operators remains open.
\end{abstract}

In the paper "Invariant subspace of strictly singular operators," which appeared in Proc. Amer. Math. Soc. 108 (1990), 931-936, the author Ji-Shou presents Theorem 1, which asserts that if $X$ is a Banach space, then every strictly singular operator in $L(X)$ is condensing. This is false. Indeed, if $T \in L(X)$ is strictly singular but not compact, then for $\alpha$ sufficiently large, $\alpha T$ is still strictly singular but is not condensing. Thus the claim that a strictly singular operator has an invariant subspace remains unproven.

Preceding the assertion of Theorem 1 is Lemma 3 which states that if $T$ is a bounded linear operator on a normed linear space $X$, and $D$ is a bounded subset of $X$ such that $T$ is $1-1$ on $D$ and $\gamma(T(D)) \geq \gamma(D)$, then $\gamma\left(T\left(D_{1}\right)\right) \geq$ $\gamma\left(D_{1}\right)$ for every subset $D_{1}$ of $D$. Here $\gamma(S)$ is the measure of noncompactness of a set $S$, that is, $\gamma(S)=\inf \left\{d \mid\right.$ there is a finite number of sets $S_{i}, i=$ $1,2, \ldots, n$, such that $S=\bigcup_{i=1}^{n} S_{i}$ and $\left.\operatorname{diam} S_{i} \leq d\right\}$. In fact, the lemma is false as seen by the following counterexample.

Let $T: l_{2} \rightarrow l_{2}$ be defined by

$$
T\left(x_{1}, x_{2}, \ldots\right)=\left(x_{1}, \frac{1}{2} x_{2}, x_{3}, \frac{1}{4} x_{4}, \ldots\right) .
$$

Take $D$ to be the unit disc and $D_{1}=\left\{x \in D \mid x_{2 n+1}=0, n=0,1, \ldots\right\}$. Now $T D_{1}$ is precompact since the operator $T_{1}: l_{2} \rightarrow l_{2}$ defined by $T_{1}\left(x_{1}, x_{2}, \ldots\right)=$ $\left(0, \frac{1}{2} x_{2}, 0, \frac{1}{4} x_{4}, \ldots\right)$ is compact. Hence $\gamma\left(T\left(D_{1}\right)\right)=0<\gamma\left(D_{1}\right)$. But $\gamma(T(D))$ $\geq \gamma(D)$. Indeed, let $D_{2}=\left\{x \in D \mid x_{2 n}=0, n=1,2, \ldots\right\}$. Since the map $J: l_{2} \rightarrow l_{2}$ defined by

$$
J\left(\alpha_{1}, \alpha_{2}, \ldots\right)=\left(\alpha_{1}, 0, \alpha_{2}, 0, \ldots\right)
$$

is an isometry and $J D=D_{2}$, we have

$$
\gamma(T(D)) \geq \gamma\left(T\left(D_{2}\right)\right)=\gamma\left(D_{2}\right)=\gamma(J D)=\gamma(D) .
$$

Department of Mathematics, University of Maryland, College Park, Maryland 20742

Received by the editors June 1, 1990 and, in revised form, June 19, 1990.

1980 Mathematics Subject Classification (1985 Revision). Primary 47A05, 47A15, 47B37.

Key words and phrases. Invariant subspace, bounded operator, normed linear spaces, singular operator. 